\title{
A highly efficient wide-band-gap host material for blue electrophosphorescent light-emitting devices
}

\author{
Dong Ryeol Whang and Youngmin You \\ Department of Materials Science \& Engineering, Seoul National University, San 56-1, Sillim 9-dong, \\ Gwanak-gu, Seoul 151-744, Korea \\ Se Hun Kim \\ Pyungtaek R\&D Center, Dongwoo FineChem Co. Ltd., 1177 Pyungtaek-Si, Kyunggi-Do, 451-764, Korea \\ Won-Ik Jeong, Young-Seo Park, Jang-Joo Kim, and Soo Young Park ${ }^{\mathrm{a})}$ \\ Department of Materials Science \& Engineering, Seoul National University, San 56-1, Sillim 9-dong, \\ Gwanak-gu, Seoul 151-744, Korea
}

(Received 8 October 2007; accepted 13 November 2007; published online 3 December 2007)

\begin{abstract}
We report on an efficient wide-band-gap host material for blue electrophosphorescence devices, namely, 1,2-trans-di-9-carbazolylcyclobutane (DCz). Photophysical studies show that lower-energy excimer formation between the carbazole units can be efficiently suppressed in a $\mathrm{DCz}$ film, thus maintaining its high triplet-state energy and inducing an exothermic energy transfer from $\mathrm{DCz}$ to iridium(III)bis[(4,6-difluorophenyl)-pyridinato- $\left.N, C^{2 \prime}\right]$ picolinate (FIrpic). Electrophosphorescent devices comprising a FIrpic:DCz emitting layer exhibit a superior performance with a maximum external quantum efficiency of $9.8 \%$, a maximum luminance efficiency of $21.5 \mathrm{~cd} / \mathrm{A}$, and a maximum power efficiency of $15.0 \mathrm{~lm} / \mathrm{W}$ at $0.01 \mathrm{~mA} / \mathrm{cm}^{2}$. (C) 2007 American Institute of Physics.

[DOI: $10.1063 / 1.2821116]$
\end{abstract}

Phosphorescent emitters, such as $\operatorname{Ir}(\mathrm{III}), \mathrm{Pt}(\mathrm{II})$, or Eu(III) complexes, are appropriate for obtaining highly efficient organic light-emitting devices (OLEDs) because they enable an effective use of both triplet and singlet excitons, thus providing a $100 \%$ internal quantum efficiency for light emission. ${ }^{1-4}$ In designing phosphorescent OLEDs, the use of a host material with a higher triplet-excited-state $\left({ }^{1} T\right)$ energy than that of a phosphorescent dopant is essential to ensure exothermic energy transfer. However, until now it has been very difficult to synthesize a suitable host material for blue phosphorescent dopants, because achieving a ${ }^{1} T$ energy higher than that of the blue phosphors is not a simple task. The reason for this is that aromatic structures are inevitably required for the host material (to ensure a good charge-carrier mobility and thermal stability), but they unfavorably lower the ${ }^{1} T$ energy. ${ }^{5}$

Carbazole is known to be a potential host material for blue electrophosphorescence because of its wide-band-gap energy, high ${ }^{1} T$ energy, and good charge-transporting ability. ${ }^{6-9}$ Particularly, arylamine-type host materials containing two carbazole groups have shown appropriately high ${ }^{1} T$ levels; for example, $N, N^{\prime}$-dicarbazolyl-3,5-benzene (mCP) exhibits a ${ }^{1} T$ energy of $2.90 \mathrm{eV}$ (Ref. 6) and 4,4'-bis(9-carbazolyl)-2-2'-dimethyl-biphenyl shows a ${ }^{1} T$ energy of $3.0 \mathrm{eV}{ }^{7}$ However, the planar and rigid arrangement of the carbazole units in these compounds leads to the formation of excimers in the concentrated film state, ${ }^{10-12}$ which results in detrimental charge trapping and the appearance of low- ${ }^{1} T$-energy sites, thus eventually limiting the device efficiency and long-term operation stability. ${ }^{5}$ Here, we propose a host material comprising a 1,2-trans-configuration of carbazoles locked in a cyclobutane ring for the suppressed excimer formation. This trans configuration prevents the in-

${ }^{a)}$ Author to whom correspondence should be addressed. FAX: 82-2-8868331. Electronic mail: parksy@snu.ac.kr. tramolecular formation of excimers between adjacent carbazoles. Furthermore, its sterically bulky nature also prevents intermolecular excimer formation-a fact that has been thoroughly investigated for photoconductors. ${ }^{13}$ From this host material, we expect a reduced concentration of excimerforming sites, thus giving a higher ${ }^{1} T$ energy and a better OLED performance. In this letter, we demonstrate the occurrence of efficient blue electrophosphorescence provided by effective exothermic energy transfer from the 1,2-trans-di-9carbazolylcyclobutane $(\mathrm{DCz})$ host to the blue phosphorescent iridium complex, iridium(III)bis[(4,6-difluorophenyl)pyridinato- $N, C^{2 \prime}$ ]picolinate (FIrpic). The obtained host material exhibited effective suppression of excimer formation, thereby providing a higher ${ }^{1} T$ level (as identified by transient photoluminescence). The OLED performance of the FIrpic:DCz device was also found to be superior to that of a reference device fabricated using $\mathrm{mCP}$ as the host material.

Figure 1(a) shows the molecular structure of $\mathrm{DCz}$, which was readily synthesized via the photochemical cyclodimerization of $N$-vinylcarbazole [acetone solution $(\sim 0.1 \mathrm{~g} / \mathrm{ml})$ under irradiation at $365 \mathrm{~nm}(4 \mathrm{~W})],{ }^{14-16}$ with a high conversion (80\% after workup).

At room temperature, the $\mathrm{DCz}$ film-evaporated on a quartz substrate-showed a characteristic carbazole monomeric emission $\left(\lambda_{\max }=353 \mathrm{~nm}\right)$ with a significantly suppressed excimer fluorescence [which commonly appears at $\lambda>420 \mathrm{~nm}$, see Fig. 1(a)]. In addition, the fluorescence spectrum of DCz overlaps very well with the metal-to-ligand charge-transfer absorption band of FIrpic so that an efficient Förster energy transfer is expected to take place from $\mathrm{DCz}$ to FIrpic. We observed a high-energy ${ }^{1} T$ emission band in the low-temperature $(12 \mathrm{~K})$ time-resolved photoluminescence spectra [see Fig. 1(a)], which suggests a facile ${ }^{1} T-{ }^{1} T$ energy transfer. Two sharp emission peaks (at 371 and $389 \mathrm{~nm}$ ), which were assigned to a delayed fluorescence, decayed rapidly, whereas a broad emission signal (at around $421 \mathrm{~nm}$, 

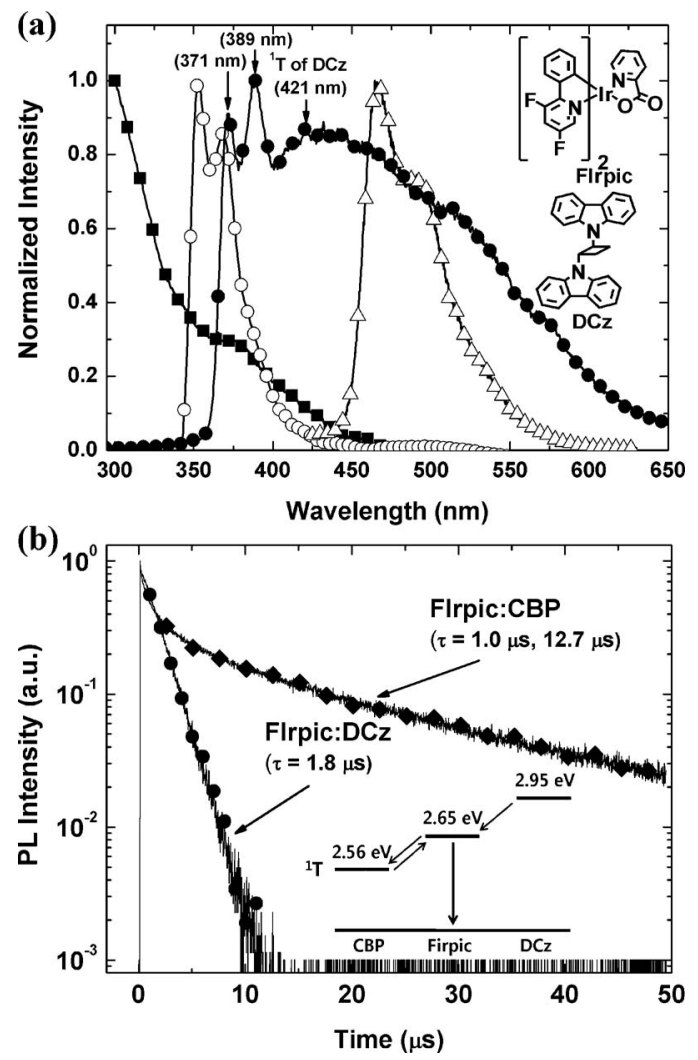

FIG. 1. Photophysical properties of emissive materials. (a) Molecular structures of FIrpic and DCz. Absorption (closed squares) and emission (open triangles) spectra of a $3 \mathrm{wt} \%$ FIrpic-doped polymethylmethacrylate (PMMA) film on a glass substrate, and fluorescence (open circles) and phosphorescence (closed circles) spectra of a DCz film (100 nm). (b) Phosphorescence decay of 6 wt \% FIrpic-doped CBP (diamonds) and DCz (circles) films.

$2.95 \mathrm{eV}$ ) was assigned to a triplet emission of DCz. Hence, considering the ${ }^{1} T$ energy of FIrpic $(2.65 \mathrm{eV})$, an exothermic energy transfer can be expected from DCz to FIrpic. To investigate this energy transfer, we measured the transient photoluminescence by means of the direct excitation of a thermally evaporated $\mathrm{DCz}$ film $(50 \mathrm{~nm})$ doped with $6 \mathrm{wt} \%$ FIrpic. 4, $4^{\prime}-N, N^{\prime}$-dicarbazole-biphenyl (CBP) and $\mathrm{mCP}$ films doped with FIrpic (6 wt \%) were also prepared and used as controls. As shown in Fig. 1(b), the phosphorescence-decay profile of FIrpic in a CBP host can be deconvoluted into two components, namely, a fast-decay component (of $1.0 \mu \mathrm{s}$ ) and a slow-decay one (of $12.7 \mu \mathrm{s}$ ). The observation of a long-lived phosphorescence indicates a thermally activated back energy transfer from FIrpic to CBP, which results from the low-lying ${ }^{1} T$ level of the latter compound. ${ }^{5}$ In contrast, the phosphorescence decay of FIrpic in $\mathrm{DCz}$ consists of a single component with a time constant of $1.8 \mu \mathrm{s}$. No longer-lived phosphorescence was observed, which indicates that the triplet excitons in the FIrpic:DCz film are efficiently trapped within FIrpic. This phosphorescence behavior is almost identical to that observed for FIrpic in the well-known high ${ }^{1} T$-energy $(2.90 \mathrm{eV})$ (Ref. 6) host $\mathrm{mCP}$ (which shows a monoexponential decay with a time constant of $1.7 \mu \mathrm{s})$. This result can be explained by an efficient exothermic energy transfer from $\mathrm{DCz}$ to FIrpic and is also consistent with a high-energy triplet emission [see Fig. 1(a)] of DCz.

Blue electrophosphorescent OLEDs have been fabricated using FIrpic:DCz and FIrpic:mCP as the emitting Downloaded 16 Mar 2008 to 147.46.94.115. Redistribution subject

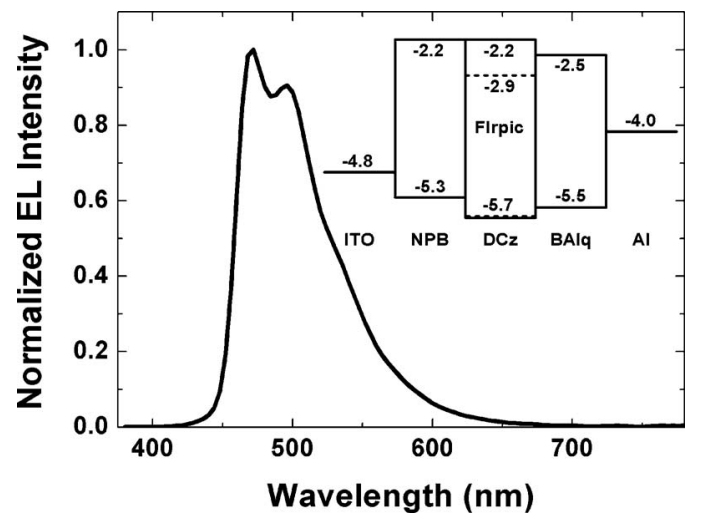

FIG. 2. EL spectra of an FIrpic:DCz device. Inset: energy diagrams of the device (in $\mathrm{eV}$ ).

layers; the devices have the configurations indium tin oxide /NPB $(400 \AA) / 8 \%$ FIrpic:DCz or mCP $(300 \AA) / \mathrm{BAlq}$ $(400 \AA) / \mathrm{LiF} \quad(10 \AA) / \mathrm{Al} \quad$ (where $\quad \mathrm{NPB}=4,4$-bis $[N$ (1-naphtyl)- $N$-phenylamino]biphenyl and $\mathrm{BAlq}=4$ biphenyloxolatoaluminum (III) bis (2-methyl-8-quinolato) 4phenylphenolate). The electroluminescence (EL) spectrum of an FIrpic:DCz device (Fig. 2, measured at a current density of $1 \mathrm{~mA} / \mathrm{cm}^{2}$ ) exhibits a characteristic FIrpic emission $\left(\lambda_{\max }=472\right.$ and $\left.496 \mathrm{~nm}\right)$ with a total absence of host emission. Figure 3 shows the current-voltage-luminance $(J-V-L)$ characteristics of FIrpic:DCz and FIrpic:mCP devices. As can be seen, the FIrpic:DCz unit maintains a higher luminance and a higher current density than its FIrpic:mCP counterpart throughout the studied voltage range (namely, $0-11 \mathrm{~V})$. It is worth noting that the FIrpic: $\mathrm{DCz}$ device is better than the FIrpic:mCP one, although both of them are exothermic-energy-transfer systems (which are quite different to the endothermic FIrpic:CBP system). The luminance of the FIrpic:DCz device reached a value of $12320 \mathrm{~cd} / \mathrm{m}^{2}$ at an applied voltage of $11 \mathrm{~V}$. This good $J-V-L$ characteristics are most likely originated from a good charge injection into the emitting layer and an efficient charge trapping within FIrpic, as shown in the energy-level diagram in the inset of Fig. 2. A maximum external quantum efficiency of $9.8 \%$, a maximum luminance efficiency of $21.5 \mathrm{~cd} / \mathrm{A}$, and a maximum power efficiency of $15.0 \mathrm{~lm} / \mathrm{W}$ were obtained (at a current density of $0.01 \mathrm{~mA} / \mathrm{cm}^{2}$ ) for our FIrpic: $\mathrm{DCz}$ device. All these characteristics are superior to those of FIrpic:mCP (which exhibits a maximum external quantum efficiency of $9.3 \%$, a maximum luminance efficiency of $20.4 \mathrm{~cd} / \mathrm{A}$, and a

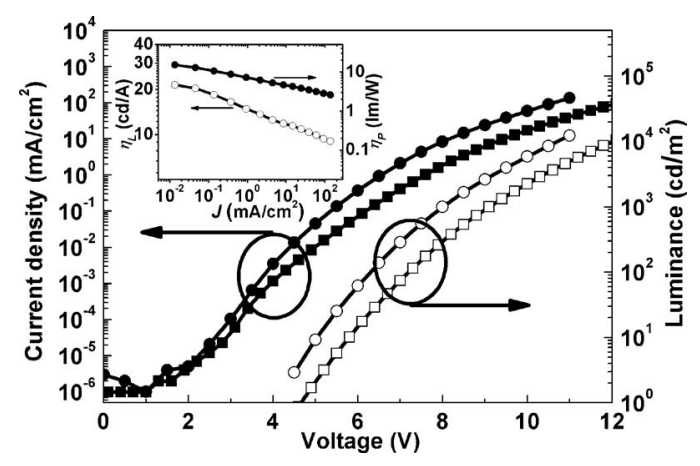

FIG. 3. $J-V-L$ characteristics of FIrpic:DCz (circles) and FIrpic:mCP (squares) devices. Inset: efficiency of the FIrpic:DCz device. 
maximum power efficiency of $13.2 \mathrm{~lm} / \mathrm{W}$ ), thus indicating an efficient exciton trapping within FIrpic.

In conclusion, we have fabricated a highly efficient blue electrophosphorescent OLED by using a carbazole-based host material DCz. Excimer formation of the carbazole units is efficiently suppressed in $\mathrm{DCz}$, which makes it possible to achieve a high ${ }^{1} T$ level of the host layer and thus exothermic energy transfer from DCz to FIrpic. As a consequence of this suppressed excimer formation, the performance of the FIrpic: $\mathrm{DCz}$ device was superior to that of a FIrpic:mCP device.

This work was partly supported by the Korea Science and Engineering Foundation (KOSEF) through the National Research Laboratory Program funded by the Ministry of Science and Technology (No. 2006-03246), and by Dongwoo FineChem Co.

${ }^{1}$ M. A. Baldo, D. F. O’Brien, Y. You, A. Shoustikov, S. Sibley, M. E. Thompson, and S. R. Forrest, Nature (London) 395, 151 (1998).

${ }^{2}$ M. A. Baldo, S. Lamansky, P. E. Burrows, M. E. Thompson, and S. R. Forrest, Appl. Phys. Lett. 75, 4 (1999).

${ }^{3}$ J. Kido, H. Hayase, K. Hongawa, K. Nagai, and K. Okuyama, Appl. Phys.
Lett. 65, 2124 (1994).

${ }^{4}$ C. Adachi, M. A. Baldo, and S. R. Forrest, J. Appl. Phys. 87, 8049 (2000).

${ }^{5}$ C. Adachi, R. C. Kwong, P. Djurovich, V. Adamovich, M. A. Baldo, M. E. Thompson, and S. R. Forrest, Appl. Phys. Lett. 79, 2082 (2001).

${ }^{6}$ R. J. Holmes, S. R. Forrest, Y.-J. Tung, R. C. Kwong, J. J. Brown, S. Garon, and M. E. Thompson, Appl. Phys. Lett. 82, 2422 (2003).

${ }^{7}$ S. Tokito, T. Iijima, Y. Suzuri, H. Kita, T. Tsuzuki, and F. Sato, Appl. Phys. Lett. 83, 569 (2003).

${ }^{8}$ G. T. Lei, L. D. Wang, L. Duan, J. H. Wang, and Y. Qiu, Synth. Met. 144, 249 (2004).

${ }^{9}$ S.-J. Yeh, M.-F. Wu, C.-T. Chen, Y.-H. Song, Y. Chi, M.-H. Ho, S.-F. Hsu, and C. H. Chen, Adv. Mater. (Weinheim, Ger.) 17, 285 (2005).

${ }^{10}$ G. E. Johnson, J. Chem. Phys. 62, 4697 (1975).

${ }^{11}$ A. Itaya, K.-I. Okamoto, and S. Kusabayashi, Bull. Chem. Soc. Jpn. 49, 2082 (1976)

${ }^{12}$ H. Benten, H. Ohkita, S. Ito, M. Yamamoto, N. Sakumoto, K. Hori, Y. Tohda, K. Tani, Y. Nakamura, and J. Nishimura, J. Phys. Chem. B 109, 19681 (2005).

${ }^{13}$ S. Tazuke, T. Inoue, S. Saito, S. Hirota, and H. Kodado, Polym. Photochem. 6, 221 (1985).

${ }^{14}$ J. W. Breitenbach, O. F. Olaj, and F. Wehrmann, Monatsch. Chem. 95, 1007 (1964)

${ }^{15}$ L. P. Ellinger, J. Feeney, and A. Ledwith, Monatsch. Chem. 96, 131 (1965).

${ }^{16}$ Y. Shirota, K. Tada, M. Shimizu, S. Kusabayashi, and H. Mikawa, Chem. Commun. 1970, 1110. 